\title{
Successful Coil Embolization of a Ruptured Basilar Artery Aneurysm in a Child with Leukemia: A Case Report
}

\author{
Shihori Hayashi, ${ }^{1}$ Taketoshi MaEHARA, ${ }^{1}$ Maki MuKaWA, ${ }^{1}$ Masaru AOYAgI, ${ }^{1}$ \\ Yoshikazu YOSHINO, ${ }^{2}$ Shigeru NEMOTO, ${ }^{2}$ Toshiaki OnO, ${ }^{3}$ and Kikuo OHNO ${ }^{1}$
}

Departments of ${ }^{1}$ Neurosurgery, ${ }^{2}$ Endovascular Surgery, and ${ }^{3}$ Pediatrics, Tokyo Medical and Dental University, Tokyo

\begin{abstract}
Ruptured intracranial aneurysms are rare in the pediatric population compared to adults. This has incited considerable discussion on how to treat children with this condition. Here, we report a child with a ruptured saccular basilar artery aneurysm that was successfully treated with coil embolization. A 12-year-old boy with acute lymphoblastic leukemia and accompanying abdominal candidiasis after chemotherapy suddenly complained of a severe headache and suffered consciousness disturbance moments later. Computed tomography scans and cerebral angiography demonstrated acute hydrocephalus and subarachnoid hemorrhage caused by saccular basilar artery aneurysm rupture. External ventricular drainage was performed immediately. Because the patient was in severe condition and did not show remarkable signs of central nervous system infection in cerebrospinal fluid studies, we applied endovascular treatment for the ruptured saccular basilar artery aneurysm, which was successfully occluded with coils. The patient recovered without new neurological deficits after ventriculoperitoneal shunting. Recent reports indicate that both endovascular and microsurgical techniques can be used to effectively treat ruptured cerebral aneurysms in pediatric patients. A minimally invasive endovascular treatment was effective in the present case, but long-term follow-up will be necessary to confirm the efficiency of endovascular treatment for children with ruptured saccular basilar artery aneurysms.
\end{abstract}

Key words: subarachnoid hemorrhage, basilar artery aneurysm, coil embolization, children, leukemia

\section{Introduction}

Intracranial aneurysms are rare in the pediatric population, with a reported prevalence between $0.5 \%$ and $4.6 \%{ }^{1}$ Children with subarachnoid hemorrhage (SAH) caused by ruptured intracranial aneurysms are even more rare. In children, treatment methods of ruptured saccular basilar artery aneurysms and long-term outcomes have not been clearly defined. Most adult cases of ruptured basilar artery aneurysms are treated endovascularly rather than microsurgically. ${ }^{1,2)}$ However, efficacy of endovascular management in children is still under discussion. On the other hand, the efficacy of microsurgery has been described in pediatric patients with cerebral aneurysms.

Here we report a child with acute lymphoblastic leukemia (ALL) and consequent systemic candida infection who suffered from a ruptured saccular basilar artery aneurysm and was successfully treated by coil embolization.

Received November 15, 2012; Accepted April 12, 2013

\section{Case Report}

A 12-year-old boy with ALL suffered from multiple liver and spleen abscesses following chemotherapy, and leukemia treatment was stopped. Almost 3 months later, he suddenly complained of a severe headache and immediately manifested consciousness disturbance. Laboratory results were as follows: white blood cell count, $12.0 \times 10^{3} / \mu \mathrm{l}$; C-reactive protein, $11.6 \mathrm{mg} / \mathrm{dl}$; aspartate aminotransferase: $222 \mathrm{U} / \mathrm{l}$; alanine aminotransferase: $144 \mathrm{U} / \mathrm{l} ; \gamma$-glutamyl transpeptidase: $108 \mathrm{U} / \mathrm{l}$; $\beta$-D-glucan: $29.5 \mathrm{pg} / \mathrm{ml}$; and candida antigen: positive. Computed tomography (CT) demonstrated diffuse SAH predominantly located in the basal cistern and enlargement of the ventricle (Fig. 1), which led to a diagnosis of SAH corresponding to Hunt \& Kosnik grade 4, World Federation of Neurosurgical Societies grade V, and Fisher group 4. Glasgow Coma Scale Score at onset was 6 (E1V1M4), and the patient did not show evidence of anisocoria or hemiparesis.

External ventricular drainage was performed immediately. Cerebrospinal fluid (CSF) cell counts increased to $522 \mu / 1$ 
due to blood cell contamination caused by SAH. Gram stain was negative, and candida was not detected. No evidence of meningitis was obtained. However possibility of bacterial or mycotic aneurysm was not completely excluded. Diffusion-weighted magnetic resonance imaging was negative for brain abscesses. Cerebral angiogram revealed a wide-necked saccular aneurysm, $7.7 \times 8.3 \times 7.0 \mathrm{~mm}$ in size, originating from the right basilar-superior cerebellar artery (SCA) junction (Fig. 2). The etiology of this aneurysm was not clearly identified. Because of poor medical condition of this patient, endovascular treatment was favored over craniotomy. The patient underwent

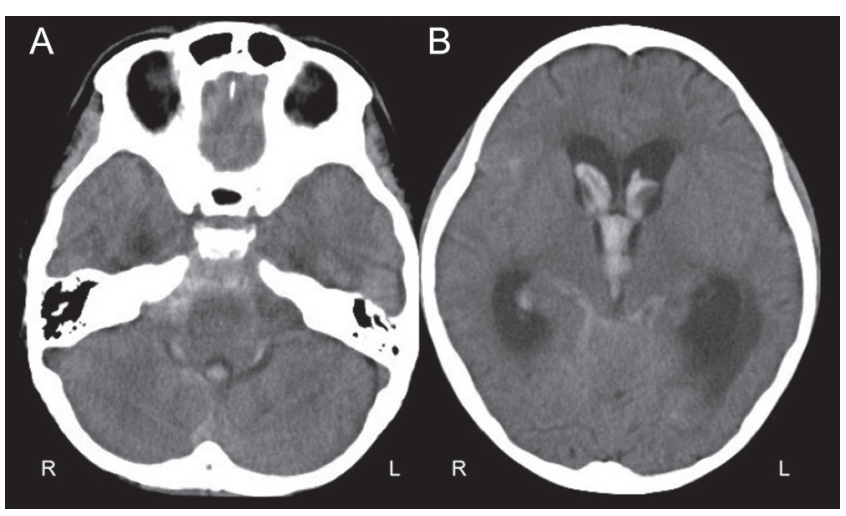

Fig. 1 Computed tomography scans on admission show subarachnoid hemorrhage in the basal cistern (A) and a small hemorrhage in the Sylvian fissure (B). Intraventricular hemorrhage with hydrocephalus was also found (B). embolization under general anesthesia using a simple technique without the use of balloon-remodeling and intravascular stent, but aneurysm sac obliteration was incomplete. Subsequent angiography revealed residual filling of the neck. We finished the coil embolization, as the preventive effect of aneurysmal re-rupture had been achieved in this case (Fig. 3).

The patient's postoperative course was uneventful, and he underwent ventriculoperitoneal shunt for normal pressure hydrocephalus 8 months after onset of SAH. Magnetic resonance angiography performed 1 year after intravascular surgery did not show recurrence of aneurysm (Fig. 4). He has not experienced any neurological symptoms and is undergoing chemotherapy for recurrent leukemia.

\section{Discussion}

Previous reports indicate that the location, size, and clinical symptoms of pediatric aneurysms are distinct from those in adults. ${ }^{3-5)}$ Intracranial aneurysms in children commonly present with $\mathrm{SAH}$; internal carotid artery bifurcations followed by middle cerebral artery bifurcations are the most common sites. The incidence of posterior circulation aneurysms and giant aneurysms is higher than adults. ${ }^{5}$ The rate of aneurysms in vertebrobasilar system comprise from $9.1 \%$ to $26 \%$ of pediatric aneurysms. ${ }^{2,4,6)}$ With regard to aneurysm shape, fusiform basilar artery aneurysms in children were much more frequent than saccular aneurysm cases (Table 1). ${ }^{3-5,7,8)}$ Among the 15 pediatric basilar artery aneurysms cases reported previously including the
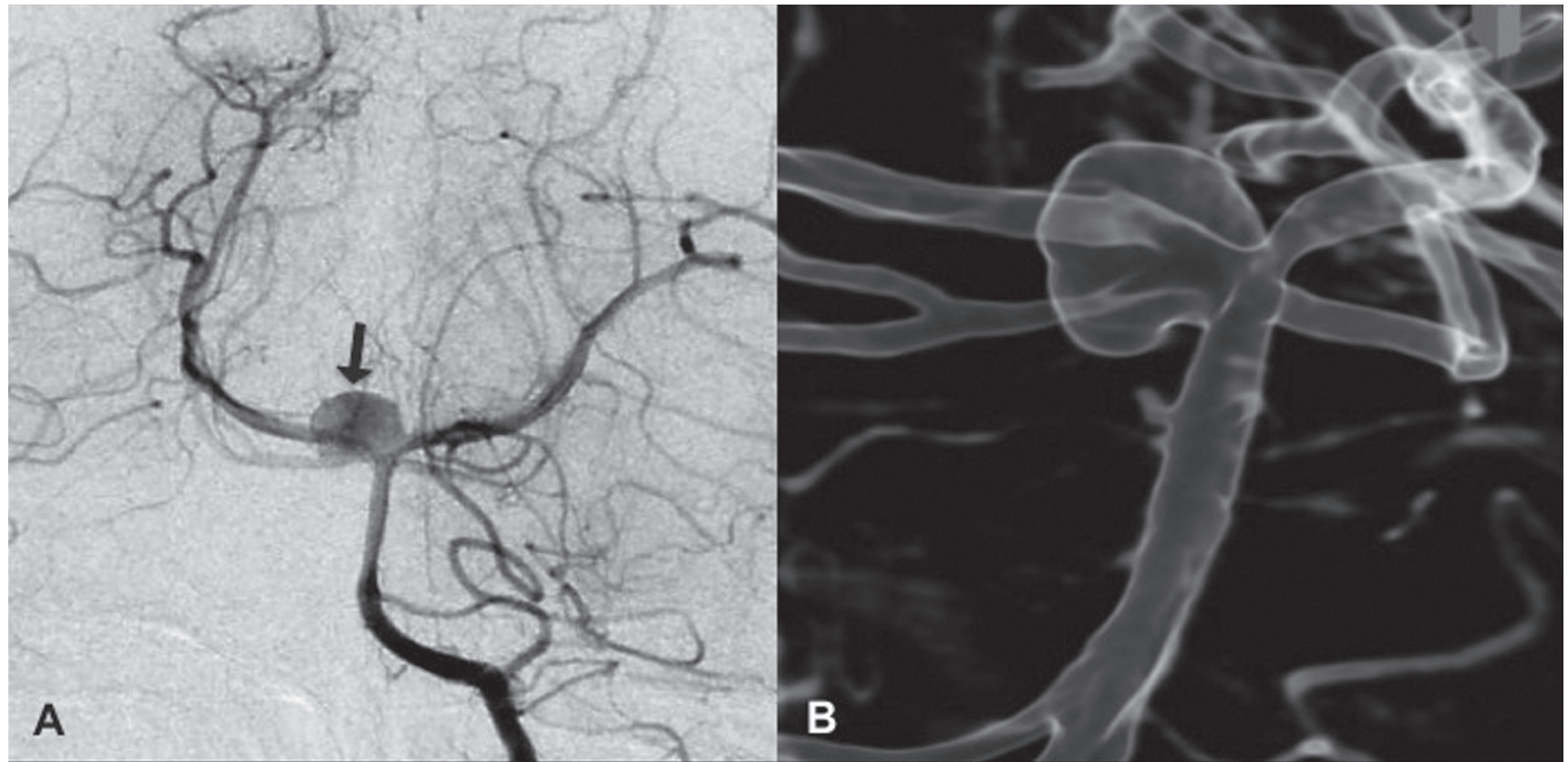

Fig. 2 Left vertebral artery angiograms show a basilar artery aneurysm originating from the right basilar-superior cerebellar artery junction (arrow). A: towne view, B: translucent 3-D image. 


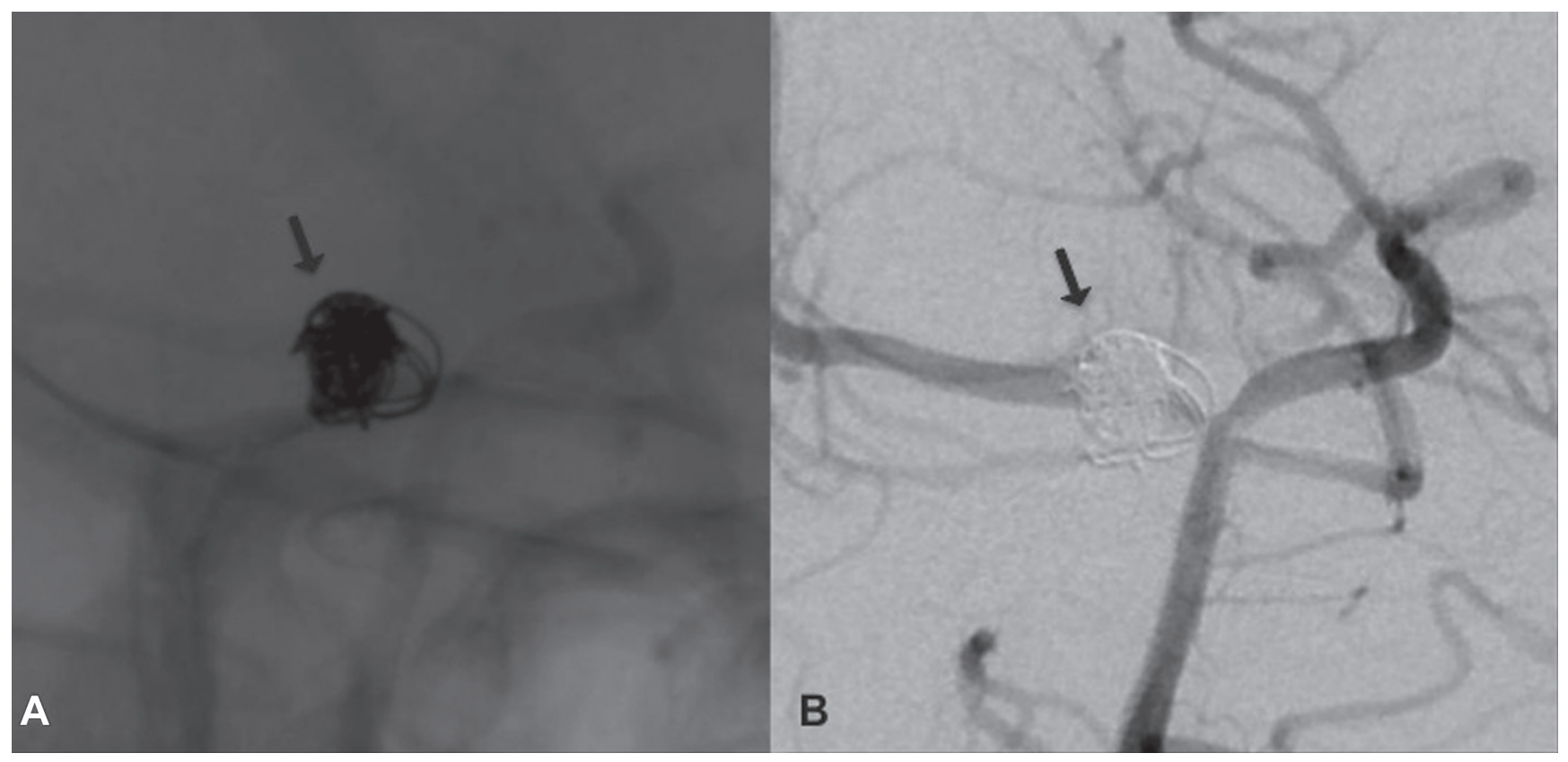

Fig. 3 A: Left vertebral artery angiograms reveal a sparse density of coil cast showing incomplete obliteration of the aneurysm sac (arrow). B: Subtracted image shows disappearance of the aneurysmal body indicating diversion of the aneurysm space from the blood stream (arrow).

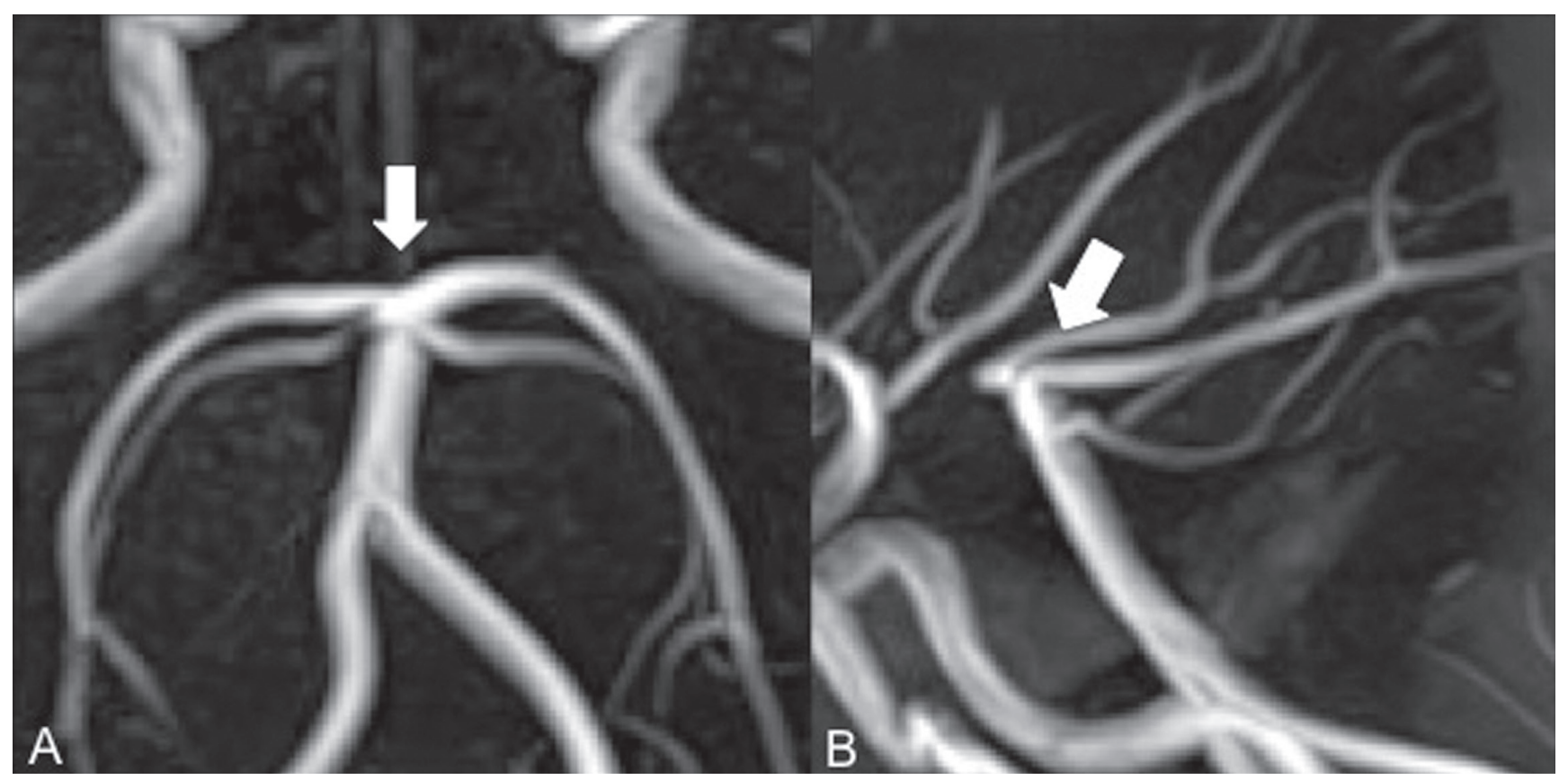

Fig. 4 Magnetic resonance angiography one year after intravascular treatment does not show recurrence of basilar artery aneurysm (arrow). A: towne view, B: lateral view.

present case, only 4 were clearly described as saccular aneurysms. Ruptured cases were observed in 8 of these 15 reported basilar artery aneurysms in children. Two of the ruptured eight aneurysms were traumatic cases which induced dissection and pseudoaneurysm.
In children, the optimal method to treat ruptured saccular basilar artery aneurysms has not been discussed in previous publications because saccular shapes were uncommon in the pediatric basilar artery aneurysms. Kakarla et al. ${ }^{8)}$ reported that 8 cases among 48 pediatric 
aneurysm patients (< 18 years old) were basilar artery aneurysms, 3 of 8 were SAH. However, there were no cases of saccular aneurysm, 6 of 8 were fusiform aneurysms and 2 were dissection and pseudoaneurysm cases by trauma (Table 1). Recent reports indicate that endovascular management has better outcomes than microsurgery for basilar artery aneurysms in adult cases. ${ }^{2,6)}$ Endovascular management of basilar artery aneurysms is also supposed to be an acceptable treatment in children. Proust et al. ${ }^{3)}$ and Stiefel et al. ${ }^{4)}$ selected endovascular intervention as treatment of ruptured basilar artery aneurysms, although their first choice for treating pediatric aneurysms was craniotomy surgery except for basilar artery aneurysms. Vaid et al. ${ }^{5)}$ employed microsurgical techniques in pediatric patients with cerebral aneurysms because children have longer life expectancies. In the analysis of the previously reported 17 cases including our patient, authors reported outcomes of 7 patients with microsurgery and 5 patients with endovascular technique. We defined that good means 5 (good recovery) and 4 (moderate disability) of glasgow outcome scale (GOS) or independent state. In the microsurgery group, 6 showed good outcome, and in the endovascular group, 4 achieved good outcome.

As far as we searched, there are no reports regarding long-term results of endovascular treatment of ruptured basilar artery aneurysm in children. According to $\mathrm{Lv}$ et al., $96 \%$ of patients with cerebral aneurysm treated endovascular surgery had good outcome (GOS score 3-5) with a period of 2 to 84 months. ${ }^{9)}$ Saraf et al. reported the similar result. ${ }^{10)}$ However in their series, none of patients of ruptured saccular basilar aneurysms were reported. There are a few reports of recanalization after coil embolization in pediatric patients. In $\mathrm{Lv}$ et al report, only 1 of 25 patients (4\%) of the embolized anterior cerebral artery aneurysm in the follow-up of 2-11 months (mean, 5.6 months) and retreated successfully. ${ }^{9}$ Pediatric cases of coiled aneurysms need results of longer-term follow-up studies including recanalization but they are not available now. Long-term follow up is necessary in children.

In our case, we selected endovascular intervention because the aneurysm was located at the basilar artery and the patient was in a serious condition. Mycotic aneurysms in children were more frequent $(10 \%)$ than those in adults (2-6\%). ${ }^{1,3)}$ Endovascular intervention may not be suitable for treatment of mycotic aneurysm cases. Although the possibility remains that systemic inflammation may have contributed to the aneurysmal formation, we could not consider that the aneurysm was mycotic. Shape and location of the aneurysm does not suggest infectious origin. There was no definite evidence of infectious aneurysm. Endovascular treatment has been applied for infectious aneurysms with satisfactory clinical results. ${ }^{11)}$

Another important issue of this case is the relationship between underlying disease, ALL, and the aneurysm development. Previous papers ${ }^{12)}$ have reported pediatric aneurysms in connective tissue disorders, including Marfan syndrome, neurofibromatosis type I, and polycystic kidney disease; congenital disorders such as cerebral arteriovenous malformation; and head injury and infectious disease. Intracranial hemorrhage (ICH) is a relatively common and often devastating complication associated with approximately $15 \%$ of leukemia patients and seems to be caused by various factors, including blood vessel wall rupture secondary to leukocyte infiltration, thrombocytopenia, platelet dysfunction, and coagulopathy. ${ }^{13)}$ Chern et al. reported that 42 patients $(55.2 \%)$ with ICH secondary to leukemia showed subdural hemorrhage, 18 $(23.6 \%)$ had intraparenchymal hemorrhage, 9 (11.9\%) had $\mathrm{SAH}$, and 7 (9.2\%) had intraventricular hemorrhage. These were all in adults, and $26 \%$ were caused by traumatic insult. ${ }^{1)}$ We were able to find only one pediatric case of SAH in a 7-year-old girl who suffered from ALL and entered remission. Seven months after the ALL onset,

Table 1 Summary of pediatric patients with basilar artery aneurysm

\begin{tabular}{|c|c|c|c|c|c|c|c|c|c|c|}
\hline \multirow{2}{*}{ Author (year) } & \multirow{2}{*}{$\begin{array}{c}\text { Number of } \\
\text { cases (SAH) }\end{array}$} & \multicolumn{3}{|c|}{ Type of aneurysm } & \multicolumn{4}{|c|}{ Treatment } & \multicolumn{2}{|c|}{ Outcome $^{\S}$} \\
\hline & & Saccular & Fusiform & $\overline{\text { Traumatic }}$ & Craniotomy & Endovascular & Both & $\overline{\text { Conservative }}$ & Good & Poor \\
\hline Proust (2001) $)^{3)}$ & $1(1)$ & \multicolumn{3}{|c|}{ Not described } & 0 & 1 & 0 & 0 & 1 & 0 \\
\hline Stiefel $(2008)^{4)}$ & $2(2)$ & \multicolumn{3}{|c|}{ Not described } & 0 & 2 & 0 & 0 & 1 & 1 \\
\hline Vaid $(2008)^{5)}$ & $2(2)$ & 2 & 0 & 0 & $\begin{array}{c}1 \\
\text { (wrapping) }\end{array}$ & 0 & 0 & 1 & 2 & 0 \\
\hline Present case & $1(1)$ & 1 & 0 & 0 & 0 & 1 & 0 & 0 & 1 & 0 \\
\hline
\end{tabular}

*Two of these 3 patients showed traumatic SAH. ${ }^{\star}$ Dissecting and pseudoaneurysm cases by trauma. ${ }^{*}$ Two patients underwent 2 surgeries. ${ }^{\S}$ Good means 5 (good recovery) and 4 (moderate disability) of Glasgow Outcome Scale (GOS) or independent state. Poor means 3 (conscious but disabled), 2 (persistent vegetative state), and 1 (death) of GOS or dependent state. 
she suddenly presented with SAH due to a ruptured aneurysm arising from the internal carotid artery at the origin of the posterior communicating artery. She underwent aneurysmal clipping and was disease-free 6 years later. The authors reported that it was not a mycotic aneurysm and there was no clear relationship between ALL and SAH. ${ }^{14)}$

In conclusion, this case report suggests that satisfactory outcome was obtained with endovascular therapy in pediatric patient with ruptured saccular basilar artery aneurysm in severe condition as well as adults. Longer follow-up is necessary to validate the efficacy and safety of endovascular surgery for pediatric patients.

\section{Conflicts of Interest Disclosure}

The authors report no conflict of interest.

\section{References}

1) Jordan LC, Johnston SC, Wu YW, Sidney S, Fullerton HJ: The importance of cerebral aneurysms in childhood hemorrhagic stroke: a population-based study. Stroke 40: 400-405, 2009

2) Ryttlefors M, Enblad P, Kerr RS, Molyneux AJ: International subarachnoid aneurysm trial of neurosurgical clipping versus endovascular coiling: subgroup analysis of 278 elderly patients. Stroke 39: 2720-2726, 2008

3) Proust F, Toussaint P, Garniéri J, Hannequin D, Legars D, Houtteville JP, Fréger P: Pediatric cerebral aneurysms. J Neurosurg 94: 733-739, 2001

4) Stiefel MF, Heuer GG, Basil AK, Weigele JB, Sutton LN, Hurst RW, Storm PB: Endovascular and surgical treatment of ruptured cerebral aneurysms in pediatric patients. Neurosurgery 63: 859-865; discussion 865-866, 2008

5) Vaid VK, Kumar R, Kalra SK, Mahapatra AK, Jain VK: Pediatric intracranial aneurysms: an institutional experience. Pediatr Neurosurg 44: 296-301, 2008

6) Molyneux A, Kerr R, Stratton I, Sandercock P, Clarke M, Shrimpton J, Holman R; International Subarachnoid Aneurysm Trial (ISAT) Collaborative Group: International
Subarachnoid Aneurysm Trial (ISAT) of neurosurgical clipping versus endovascular coiling in 2143 patients with ruptured intracranial aneurysms: a randomised trial. Lancet 360: 1267-1274, 2002

7) Huang J, McGirt MJ, Gailloud P, Tamargo RJ: Intracranial aneurysms in the pediatric population: case series and literature review. Surg Neurol 63: 424-432; discussion 432-433, 2005

8) Kakarla UK, Beres EJ, Ponce FA, Chang SW, Deshmukh VR, Bambakidis NC, Zabramski JM, Spetzler RF: Microsurgical treatment of pediatric intracranial aneurysms: long-term angiographic and clinical outcomes. Neurosurgery 67: 237-249; discussion 250, 2010

9) Lv X, Jiang C, Li Y, Yang X, Wu Z: Endovascular treatment for pediatric intracranial aneurysms. Neuroradiology 51: 749-754, 2009

10) Saraf R, Shrivastava M, Siddhartha W, Limaye U: Intracranial pediatric aneurysms: endovascular treatment and its outcome. Clinical article. J Neurosurg Pediatrics 10: 230-240, 2012

11) Chun JY, Smith W, Halbach VV, Higashida RT, Wilson CB, Lawton MT: Current multimodality management of infectious intracranial aneurysms. Neurosurgery 48: 1203-1213; discussion 1213-1214, 2001

12) McDonald RL. Surgical management of aneurysms in the pediatric age group. in Welch KM, Caplan LR, Reis DJ, Siesjo BK, Weir B (eds): Primer on Cerebrovascular Diseases. New York, Academic Press, 1997, pp 484-487

13) Chern JJ, Tsung AJ, Humphries W, Sawaya R, Lang FF: Clinical outcome of leukemia patients with intracranial hemorrhage. Clinical article. J Neurosurg 115: 268-272, 2011

14) Ehrlich ME, Lavyne MH: Aneurysmal subarachnoid hemorrhage in a child with leukemia. Surg Neurol 30: 144-147, 1988

Address reprint requests to: Shihori Hayashi, MD, Department of Neurosurgery, Tokyo Medical and Dental University, 1-5-45, Yushima, Bunkyo-ku, Tokyo 113-8519, Japan. e-mail: hayansrg@tmd.ac.jp 